\title{
Search for resonances in top anti-top pairs with the ATLAS detector
}

\author{
Johannes Erdmann* \\ Yale University \\ E-mail: johannes.erdmannecern.ch \\ On behalf of the ATLAS Collaboration
}

Four analyses searching for resonant production of top quark pairs using data taken with the ATLAS detector at the CERN Large Hadron Collider are reviewed. Top anti-top pairs decaying in the dileptonic, all-hadronic and lepton+jets channels were studied using resolved as well as boosted regimes. Moreover, resonant top+jet production was searched for. Up to $4.7 \mathrm{fb}^{-1}$ of data taken in proton-proton collisions at a centre-of-mass energy of $7 \mathrm{TeV}$ were analysed. All data were found to be consistent with the Standard Model. Hence, upper limits were set on the production cross-section times branching ratio into top anti-top pairs for a potential resonance, as well as on the minimal mass of such a resonance in narrow and large width benchmark scenarios.

Xth Quark Confinement and the Hadron Spectrum

8-12 October 2012

TUM Campus Garching, Munich, Germany

\footnotetext{
* Speaker.
} 


\section{Introduction}

In several models beyond the Standard Model (SM), new particles decaying to top anti-top pairs $(t \bar{t})$ are predicted. In this overview, searches for such a resonance using data taken with the ATLAS detector [1] at the CERN Large Hadron Collier (LHC) are reviewed. All decay channels of $t \bar{t}$ were explored in order to increase the sensitivity of the searches: the dileptonic, the all-hadronic and the lepton+jets channel. When top quarks are produced with large boosts, their decay products tend to be collimated and overlap. This is called the boosted regime as opposed to the resolved regime. The decay of hadronically decaying top quarks may then result in one large jet with large invariant mass (fat jet). In the all-hadronic channel as well as in the lepton+jets channel, the boosted regime was exploited to enhance the sensitivity of the searches.

Two benchmark models predicting a resonance with a small and a large width, respectively, were chosen for the interpretation of the $t \bar{t}$ resonance searches: in models with strong electroweak symmetry breaking through top quark condensation [2], a leptophobic topcolour $Z^{\prime}$ boson with a relative width of $1.2 \%$ is predicted for a specific choice of model parameters [3]. In RandallSundrum models with warped extra dimensions [4], Kaluza-Klein (KK) gluons $g_{K K}$ with a width of $15.3 \%$ are predicted for left-handed $g_{L}$ and right-handed couplings $g_{R}$ of $g_{L}=g_{R}=-0.2 g_{S}$ for light quarks, $g_{L}=g_{S}$ and $g_{R}=-0.2 g_{S}$ for $b$-quarks, and $g_{L}=g_{S}$ and $g_{R}=4 g_{S}$ for top quarks, with $g_{S}=\sqrt{4 \pi \alpha_{S}}$.

The measurement of the charge asymmetry in $t \bar{t}$ production at the Tevatron [5] shows some tension with the SM. One possible explanation of the discrepancy are models with top-flavour violating processes which predict the existence of a colour-single $W^{\prime}$ or a colour-octet $\phi$ decaying to a top quark and another up-type quark: $W^{\prime} \rightarrow \bar{t} q$ and $\phi \rightarrow t q$, respectively [6]. At the LHC, such a resonance would be produced in association with another (anti-)top quark leading to a $t \bar{t}+$ jets signature and a $t+$ jet and $\bar{t}+$ jet resonance, respectively. In the analysis presented here, top + jet resonances were searched for in the lepton+jets channel in the resolved regime.

Searches for $t \bar{t}$ resonances have been carried out in $p \bar{p}$ collisions at the D0 [7] and CDF experiments [8] as well as in $p p$ collisions at $\sqrt{s}=7 \mathrm{TeV}$ at the CMS experiment [9]. Data were found to be consistent with the SM and lower limits were set at 95\% CL on the mass of $Z^{\prime}$ and $g_{K K}$ resonances of $1.49 \mathrm{TeV}$ and $1.82 \mathrm{TeV}$, respectively [9]. Searches for top+jet resonances carried out at the CDF experiment [10] yielding lower limits of $300 \mathrm{GeV}$ and $200 \mathrm{GeV}$ for $W^{\prime}$ and $\phi$ masses, respectively. At the CMS experiment, a search for top+jet resonances [11] resulted in a lower limit of $840 \mathrm{GeV}$ for the mass of a $W^{\prime}$, with a right-handed coupling of $g_{R}=2 g_{S}$.

The data analysed in the analyses presented here were taken with the ATLAS detector in proton-proton collisions in 2011 at $\sqrt{s}=7 \mathrm{TeV}$. While the analysis in the dileptonic channel used $2.05 \pm 0.08 \mathrm{fb}^{-1}$ [12] of data, the other searches used the full dataset of $4.7 \pm 0.2 \mathrm{fb}^{-1}$. Events were selected with single lepton triggers in the dileptonic and resolved lepton+jets analyses, as well as with jet triggers in the boosted analyses.

For the modelling of the different signal and background processes, Monte Carlo (MC) simulated events were produced using dedicated generators and a full detector simulation [13] based on Geant4. Simulated events were reconstructed using the same reconstruction software as used for data. All simulated samples were reweighted to represent the same distribution of the number of interactions per event as observed in data. 
The multijet background in the all-hadronic analysis was estimated from data. Also in the other analyses, part of the background processes were estimated from data. Where MC simulations were used and not normalised to a control region in data, most precise estimates of the cross-sections of the processes in question were used. In particular, the $t \bar{t}$ production cross-section was calculated in approximate next-to-next-to-leading order [14].

The $Z$ ' signal was modelled using PYTHIA for a variety of mass points and normalised to the next-to-leading order prediction in Ref. [15]. The $g_{K K}$ signal was modelled using MADGRAPH and the parton shower was added using PYTHIA. The cross-section for $g_{K K}$ production was calculated using PYTHIA. The top+jet resonance signal was generated using MADGRAPH interfaced to PYTHIA. $W^{\prime}$ production was normalised to a next-to-leading-order calculation [16], while $\phi$ production was normalised to a leading-order calculation [6].

Electron candidates [17] were constructed from electromagnetic cluster with an associated track. Electrons had to have a $E_{\mathrm{T}}>25 \mathrm{GeV}$, had to be within $|\eta|<1.37$ or $1.52<|\eta|<2.37$, and had to be isolated from other activity. Muon candidates [18] were constructed from combined tracks in the inner detector and in the muon spectrometer within $|\eta|<2.5$. Muons had to have a $p_{\mathrm{T}}>20 \mathrm{GeV}$, and had to be isolated from other activity. In case of the boosted analyses, energydependent cone sizes were used for the lepton isolation criteria (mini-isolation). Jets [19] were reconstructed using the anti- $k_{t}$ algorithm with a radius parameter of 0.4 . Jets were required to have a $p_{\mathrm{T}}>25 \mathrm{GeV}$ and to be within $|\eta|<2.5$. Jets were $b$-tagged using multivariate tagging algorithms [20]. Operating points with efficiencies of $60 \%$ and $70 \%$ and mistagging probabilities less than $1 \%$ were used. For the boosted analysis in the lepton+jets (all-hadronic) channel, fat jets were reconstructed using the anti- $k_{t}$ (Cambridge/Aachen) algorithm with a radius parameter of 1.0 (1.5), requiring $p_{\mathrm{T}}>350 \mathrm{GeV}(200 \mathrm{GeV})$ and $|\eta|<2.0(2.5)$.

\section{Search for top anti-top resonances in the dileptonic channel}

For the search for $t \bar{t}$ resonances in the dileptonic channel [21], events were selected requiring two leptons with opposite charge and two or more jets. In the ee and $\mu \mu$ channels, events with $\left|m_{Z}-m_{l l}\right|<10 \mathrm{GeV}$, with $E_{\mathrm{T}}<40 \mathrm{GeV}$ or with $m_{l l}<10 \mathrm{GeV}$ were discarded to reduce background contributions from $Z$ boson and meson resonances. In the $e \mu$ channel, the scalar sum of the $p_{\mathrm{T}}$ of leptons and jets $\left(H_{T}\right)$ was required to be larger than $130 \mathrm{GeV}$ in order to reduce background from other sources than $t \bar{t}$ production.

Background contributions from $t \bar{t}$, single top and diboson productions were estimated using MC simulated events and theoretical predictions for the cross sections. For $W+$ jets and $Z+j e t s$ productions, the MC samples were normalised to data exploiting the charge asymmetry of $W$ boson production and the enhancement of the $Z$ boson background in events with $m_{l l}$ close to the $Z$ boson mass. The contribution from QCD multijet production was estimated using the matrix method [22].

The two neutrinos in the final state render the reconstruction of the invariant $t \bar{t}$ mass impossible. However, the sum of $H_{T}$ and $Z_{\mathrm{T}}$ is strongly correlated with the $t \bar{t}$ invariant mass and was chosen to discriminate a possible signal from the various background contributions.

Fig. 1 shows the distribution of $H_{T}+Z_{\mathrm{T}}$ for signal and background expectations as well as for data. No excess above the background was observed and 95\% confidence level (CL) upper limits were set on the $g_{K K}$ production cross section times branching ratio into $t \bar{t} \sigma \times \mathrm{BR}\left(g_{K K} \rightarrow t \bar{t}\right)$ 
(Fig. 2). Various sources of systematic uncertainties were evaluated. No systematic source was found to dominate the overall uncertainty. The combination of all systematic uncertainties, however, changed the limits on the $g_{K K}$ cross section by factors of $1.5-3.0$ depending on the mass: $g_{K K}$ masses between 500 and $1080 \mathrm{GeV}$ were excluded, in agreement with the expected limits.

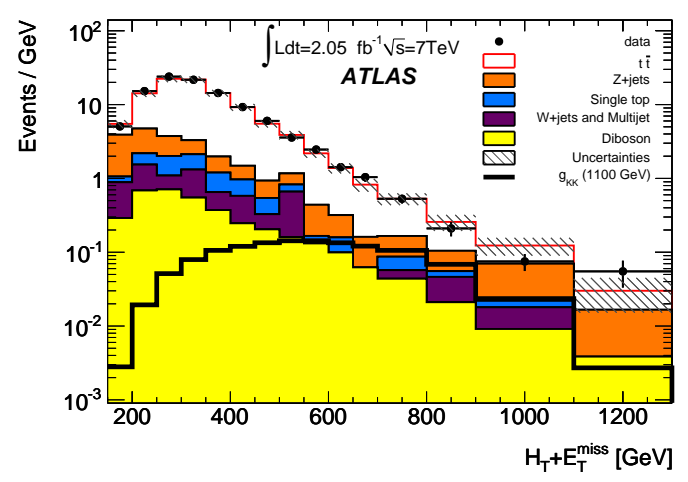

Figure 1: Distribution of $H_{T}+Z_{\mathrm{T}}$ for the SM background contributions and for data in the dilepton channel [21]. A potential signal from a 1.1 TeV KK gluon is also overlaid.

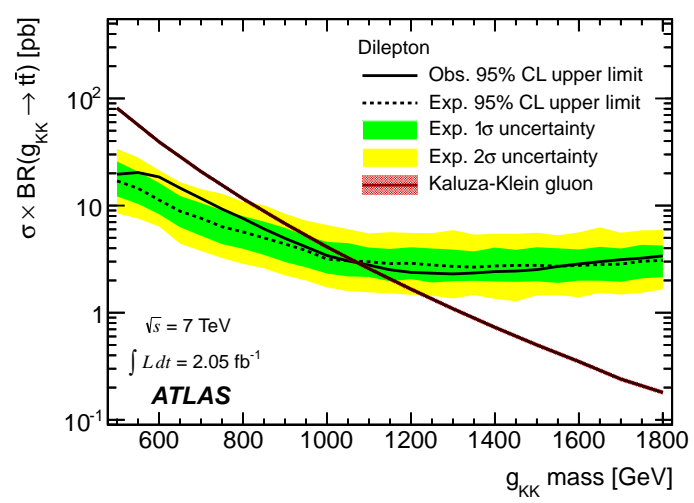

Figure 2: $95 \% \mathrm{CL}$ upper limits on $\sigma \times \mathrm{BR}$ in the dilepton channel [21]. The predicted $\sigma \times \mathrm{BR}$ is also overlaid.

\section{Search for top anti-top resonances in the all-hadronic channel}

For the search for $t \bar{t}$ resonances in the all-hadronic channel [23], events were selected with at least two fat jets with $p_{\mathrm{T}}>200 \mathrm{GeV}$. In order to suppress background contributions from QCD multijet production, both jets had to be qualified as top-like (top-tagged) by the HEPTopTagger algorithm [24]. This algorithm exploits the substructure of fat jets originating from the hadronic decay of boosted top quarks by identifying three subjets, removing contributions from pile-up and requiring the invariant mass of the subjet system to be compatible with the mass of the top quark. Additionally, both jets were required to be $b$-tagged by matching a small-radius jet to the fat jet within a cone of 1.4 in $\eta$ - $\phi$-space. Events with isolated electrons or muons were rejected.

The dominant background contributions from $t \bar{t}$ and QCD multijet production were estimated using control regions in data by reducing the number of $b$ - and top-tags. The $t \bar{t} \mathrm{MC}$ simulation was normalised using the region with only one top-tag and at least $2 b$-tags. QCD multijet production was estimated combining the control regions to extrapolate to the signal region exploiting the fact that top- and $b$-tagging are uncorrelated for this background.

No excess with respect to the SM background expectations were found in the $t \bar{t}$ invariant mass spectrum (Fig. 3), and 95\% CL upper limits were set on $\sigma \times \mathrm{BR}$ for the production of a $Z^{\prime}$ boson (Fig. 4) and a KK gluon. Various sources of systematic uncertainties were evaluated, and the dominant sources were found to be the modelling of the $b$-tagging performance and the jet energy scale (JES), and the normalisation uncertainty of the irreducible $t \bar{t}$ background. $Z^{\prime}$ bosons with masses in the ranges $0.70-1.00 \mathrm{TeV}$ and $1.28-1.32 \mathrm{TeV}$ were excluded as well as $\mathrm{KK}$ gluons with masses between 0.7 and $1.48 \mathrm{TeV}$, in agreement with the expected limits. 


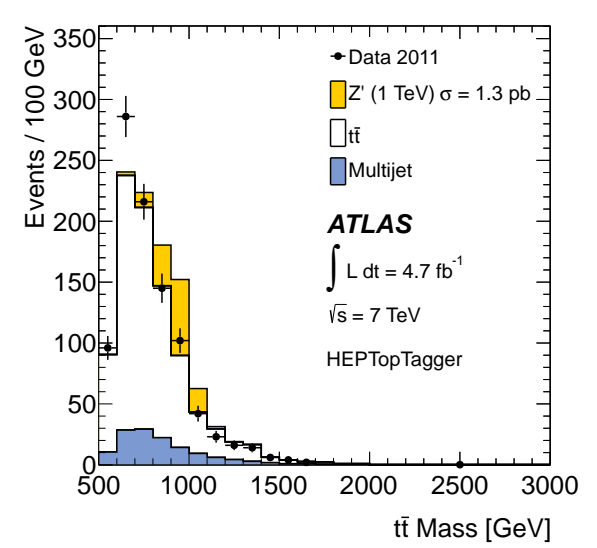

Figure 3: Distribution of the $t \bar{t}$ invariant mass for the SM background processes and for data in the all-hadronic channel [23]. A potential signal from a $1 \mathrm{TeV} Z^{\prime}$ boson is also overlaid.

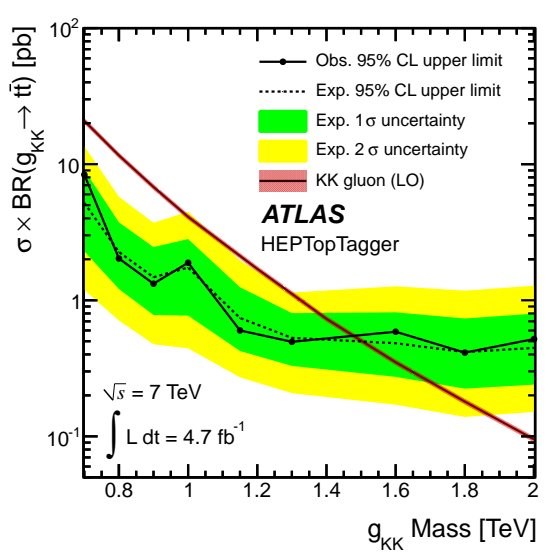

Figure 4: 95\% CL upper limits on $\sigma \times \mathrm{BR}$ for the production of a $Z^{\prime}$ boson in the all-hadronic channel [23]. The predicted values for $\sigma \times \mathrm{BR}$ are also overlaid.

\section{Search for top anti-top resonances in the lepton+jets channel}

The search in the lepton+jets channel [25] is an update of previous searches using $2.05 \mathrm{fb}^{-1}$ of data in the resolved [21] and the boosted regime [26]. The resolved and the boosted parts of the analysis were based on a common preselection: a lepton fulfilling a mini-isolation criterion had to be present in the event. In order to suppress contributions from QCD multijet production, in the electron channel, $\mathbb{Z}_{\mathrm{T}}$ had to be larger than $35 \mathrm{GeV}$ and $m_{T}^{1}$ had to be larger than $25 \mathrm{GeV}$. In the muon channel, the following requirements were made: $\not_{\mathrm{T}}>20 \mathrm{GeV}$ and $\not_{\mathrm{T}}+m_{T}>60 \mathrm{GeV}$.

In the resolved analysis, at least three jets were required. If none of the jets had an invariant mass of at least $60 \mathrm{GeV}$, interpreted as being due to the merging of two jets, an additional fourth jet had to be present in the event. At least one of these jets had to be $b$-tagged. The invariant mass of the $t \bar{t}$ system was reconstructed from the jets which had the lowest $\chi^{2}$ value out of all jet permutations. The $\chi^{2}$ function exploited the decay topology of the lepton+jets decay of $t \bar{t}$ with the invariant mass constraints at the decay vertices. The four-momentum of the neutrino was estimated using the $E_{\mathrm{T}}$ and the invariant mass of the leptonically decaying $W$ boson.

In the boosted analysis, at least one fat jet with $p_{\mathrm{T}}>350 \mathrm{GeV}$ and an invariant mass of at least $100 \mathrm{GeV}$ was required. The first $k_{t}$ splitting scale $\sqrt{d_{12}}$ [27] had to be larger than $40 \mathrm{GeV}$ to reduce background contributions from QCD multijet production. One small-radius jet had to be closer to the lepton than 1.5 in $\eta-\phi$ space. Moreover, one small-radius jet in the event had to be $b$-tagged. Cuts were applied in $\eta-\phi$ space to ensure that the fat jet and the leptonic hemisphere of the event were well separated from each other. The invariant $t \bar{t}$ mass was constructed from the fourmomenta of these objects and the neutrino, which was estimated as done in the resolved analysis. Events fulfilling the boosted selection were removed from the resolved analysis.

The contributions from $t \bar{t}$, single top, $Z+$ jets and diboson productions were estimated using MC simulations and theoretical cross section calculations. The normalisation of the $W+$ jets contri-

\footnotetext{
${ }^{1} m_{T}=\sqrt{2 p_{\mathrm{T}} E_{\mathrm{T}}(1-\cos \Delta \phi)}$, with $p_{\mathrm{T}}$ the transverse momentum of the charged lepton and $\Delta \phi$ the azimuthal angle between the charged lepton and $\mathbb{F}_{\mathrm{T}}$.
} 
bution was taken from data using the charge asymmetry in $W+$ jets events. In order to increase the number of $W+$ jets events in the boosted analysis, the requirements on the fat jet were loosened. The contribution from QCD multijet production was estimated from data using the matrix method and was validated in multijet enhanced control regions for the resolved as well as the boosted selection.

The spectrum of the invariant $t \bar{t}$ mass was found to be consistent with the expectation from SM background processes (Fig. 5) and 95\% confidence level limits were set on $\sigma \times$ BR for the production of a $Z^{\prime}$ boson and a KK gluon (Fig. 6), respectively. Various sources of systematic uncertainties were evaluated, and the dominant sources were found to be the modelling of the JES, the $b$-tagging performance and the modelling uncertainty of the $t \bar{t}$ background.

Combining the resolved and the boosted analysis parts resulted in improved limits, the resolved analysis being more sensitive for lower invariant $t \bar{t}$ masses and the boosted analysis being more sensitive for larger masses. $Z^{\prime}$ bosons with masses between 0.5 and $1.7 \mathrm{TeV}$ were excluded as well as KK gluons with masses between 0.7 and $1.9 \mathrm{TeV}$, in agreement with the expected limits.

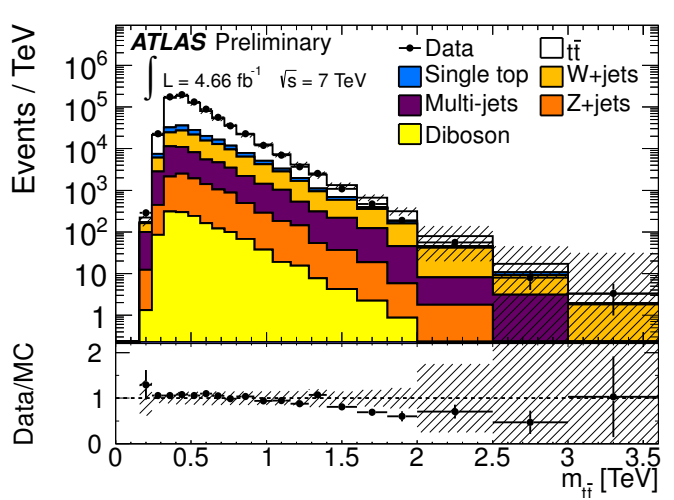

Figure 5: Distribution of the $t \bar{t}$ invariant mass for the various SM background processes as well as for data in the lepton+jets channel [25].

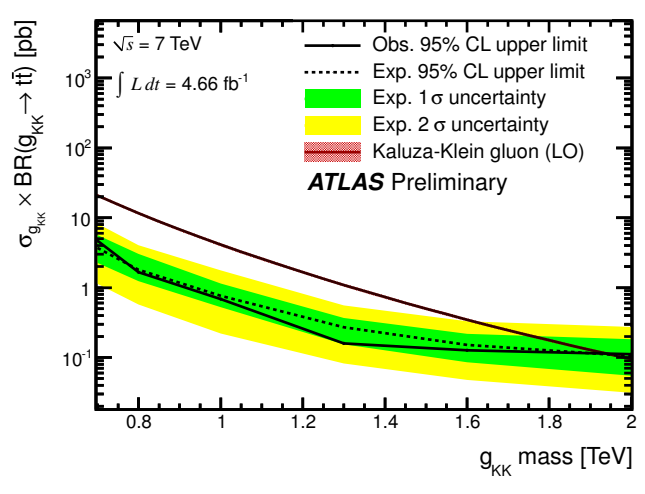

Figure 6: $95 \% \mathrm{CL}$ upper limits on $\sigma \times \mathrm{BR}$ for the production of a KK gluon in the lepton+jets channel [25]. The predicted values for $\sigma \times \mathrm{BR}$ are also overlaid.

\section{Search for top+jet resonances}

For the search of top+jet resonances [28], one lepton and at least five jets were required, out of which one had to be $b$-tagged. In the electron channel, $\not_{\mathrm{T}}$ as well as $m_{T}$ had to be larger than $30 \mathrm{GeV}$. In the muon channel, $\mathbb{E}_{\mathrm{T}}$ had to be larger than $20 \mathrm{GeV}$ and $\not_{\mathrm{T}}+m_{T}$ had to be larger than $60 \mathrm{GeV}$. The topology of the $t \bar{t}$ decay was reconstructed using a kinematic fitter, which exploited the decay topology, in particular the invariant masses at the decay vertices, resulting in four jets associated best with the $t \bar{t}$ decay. The remaining jets were used to build the maximum (anti)top+jet masses $m_{t j}$ and $m_{\bar{t} j}$, which provide discrimination between signal and the SM background processes dominated by SM $t \bar{t}$ production. The same jet could be used for both observables.

SM background processes include $t \bar{t}, W+$ jets, $Z+$ jets, single top and diboson productions, which were modelled using MC simulations and theory calculations for the cross sections. The small background from QCD multijet events was modelled from data. Various control regions 
were defined with enhanced contributions from the main backgrounds, $W+$ jets and $t \bar{t}$ productions, also making sure that the $t \bar{t}$ background was modelled well in the four as well as in the five jet bin.

The SM background processes were found to be well under control and in the signal region no excess over the SM prediction was found in the $m_{t j}$ (Fig. 7) and $m_{\bar{t} j}$ distributions. Various sources of systematic uncertainties were evaluated, and the dominant systematic sources were found to be the modelling of the JES, the $b$-tagging performance, and the normalisation of the SM background processes. Hence, upper 95\% CL limits were set on the production cross section of $W^{\prime}$ and $\phi$ bosons, which were also interpreted as lower limits of $430 \mathrm{GeV}$ on the masses of these bosons assuming a right-handed coupling $g_{R}$ of 1 , in agreement with the expected limits. These limits increase to $1.10 \mathrm{TeV}(1.45 \mathrm{TeV})$ for a $W^{\prime}(\phi)$ boson when increasing $g_{R}$ to 2 . Thus, this search represents the current most stringent limits on these models. Moreover, Fig. 8 shows the limits in the $g_{R}-m$ plane for the $W^{\prime}$ production, indicating that the largest part of the phase space preferred by the Tevatron charge asymmetry measurements is excluded by this analysis. The same holds true for the production of a $\phi$ boson.

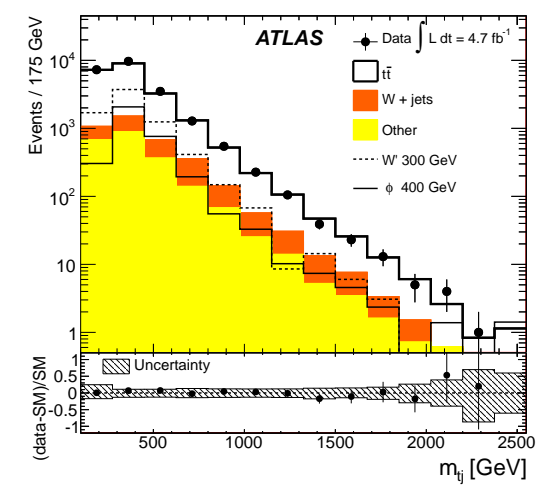

Figure 7: Distribution of the top+jet invariant mass $m_{t j}$ for the SM background processes as well as for data [28]. Potential signals for a $W^{\prime}$ and a $\phi$ boson with masses of $300 \mathrm{GeV}$ and $400 \mathrm{GeV}$, respectively, are also overlaid.

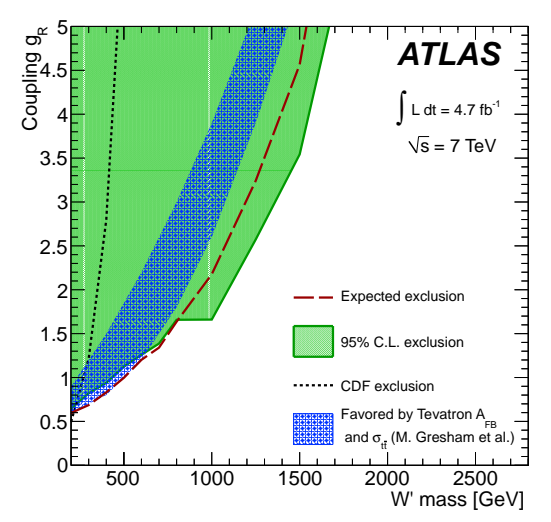

Figure 8: $95 \%$ CL upper limits on $\sigma$ for the production of a $W^{\prime}$ as a function of the coupling $g_{R}$ and the particle mass [28]. The result of the CDF search [10] and the region favoured by the Tevatron charge asymmetry measurement are also shown.

\section{Summary}

Searches for resonances in $t \bar{t}$ production were presented exploiting all $t \bar{t}$ decay channels as well as resolved and boosted topologies. No discrepancies with respect to the SM were found and $95 \%$ CL upper limits on the cross section times branching ratio into $t \bar{t}$ were set in benchmark scenarios with a narrow-width $Z^{\prime}$ boson and a large-width KK gluon. When interpreting these limits as lower mass limits, $Z^{\prime}$ bosons and KK gluons were excluded with masses below 1.7 and $1.9 \mathrm{TeV}$, respectively. These limits are stronger than the limits obtained by other experiments so far $[7,8,9]$. Moreover, a search for colour-singlet $W^{\prime}$ and colour-octet $\phi$ bosons was presented. No discrepancy with respect to the SM was found and 95\% CL upper limits were set on the production cross sections of such particles yielding the most stringent lower bounds on their masses. 


\section{References}

[1] ATLAS Collaboration, JINST 3 (2008) S08003.

[2] C. T. Hill, Phys. Lett. B345 (1995) 483 [hep-ph/ 9411426 ].

[3] R. M. Harris et al., arXiv: hep-ph/9911288 (1999).

[4] K. Agashe et al., Phys. Rev. D77 (2008) 015003 [hep-ph / 0612015$].$

B. Lillie et al., JHEP 0709 (2007) 074 [hep-ph / 0701166 ].

B. Lillie et al., Phys. Rev. D76 (2007) 115016 [0 066 . 3960].

[5] CDF Collaboration, submitted to Phys. Rev. D [1211.1003]. DØ Collaboration, Phys. Rev. D84 (2011) 112005 [1107 . 4995].

[6] M. I. Gresham et al., Phys. Rev. D84 (2011) 034025 [1102 . 0018]. Y. Cui et al., JHEP 1107 (2011) 127 [11 06 . 3086]. S. Knapen et al., Phys. Rev. D86 (2012) 014013 [1111 . 5857].

[7] DØ Collaboration, Phys. Rev. D85 (2012) 051101 [1111. 1271].

[8] CDF Collaboration, Phys. Rev. D84 (2011) 072004 [1107. 5063]. CDF Collaboration, Phys. Rev. D84 (2011) 072003 [1108 . 4755].

[9] CMS Collaboration, JHEP 1209 (2012) 029 [1204 . 2488].

CMS Collaboration, submitted to JHEP [1209.4397].

CMS Collaboration, submitted to Phys. Rev. D [1211.3338].

[10] CDF Collaboration, Phys. Rev. Lett. 108 (2012) 211805 [1203 . 3894].

[11] CMS Collaboration, Phys. Lett. B717 (2012) 351 [1206.3921].

[12] ATLAS Collaboration, ATLAS-CONF-2011-116, https://cds.cern.ch/record/1376384. ATLAS Collaboration, Eur. Phys. J. C71 (2011) 1630 [1101.2185].

[13] ATLAS Collaboration, Eur. Phys. J. C70 (2010) 823 [1005 . 4568].

[14] S. Moch and P. Uwer, Phys. Rev. D78 (2008) 034003 [0 804 . 1476].

M. Aliev et al., Comput. Phys. Commun. 182 (2011) 1034 [1007.1327].

[15] R. M. Harris and S. Jain, Eur. Phys. J. C72 (2012) 2072 [1112 . 4928].

J. Gao et al., Phys. Rev. D82 (2010) 014020 [10 04 . 0876].

[16] J. Adelman et al., submitted to JHEP [1206.5731].

[17] ATLAS Collaboration, Eur. Phys. J. C72 (2012) 1909 [1110 . 3174].

[18] ATLAS Collaboration, ATLAS-CONF-2011-063, https://cds.cern.ch/record/1345743.

[19] ATLAS Collaboration, arXiv:1112.6426 (2011).

[20] ATLAS Collaboration, ATLAS-CONF-2012-043, https://cds.cern. ch/record/1435197.

[21] ATLAS Collaboration, Eur. Phys. J. C72 (2012) 2083 [1205 . 5371].

[22] ATLAS Collaboration, Phys. Lett. B707 (2012) 459 [1108 . 3699].

[23] ATLAS Collaboration, submitted to JHEP [1211.2202].

[24] T. Plehn et al., JHEP 1010 (2010) 078 [1006 . 2833].

[25] ATLAS Collaboration, ATLAS-CONF-2012-136, https://cds.cern.ch/record/1478974.

[26] ATLAS Collaboration, JHEP 1209 (2012) 041, [1207. 2409 ].

[27] ATLAS Collaboration, ATLAS-CONF-2012-065, https://cds.cern.ch/record/1459530.

[28] ATLAS Collaboration, Phys. Rev. D86 (2012) 091103 [1209. 6593]. 